\title{
Advantages of modified drug delivery system in psychiatry
}

\author{
Anant D. Patil*
}

Freelance consultant: Medical communication and training

Received: 12 June 2013

Accepted: 28 June 2013

\section{*Correspondence to:}

Dr. Anant D. Patil,

Email: anantdpatil@gmail.com

\section{(C) 2013 Patil AD. This is an} open-access article distributed under the terms of the Creative Commons Attribution License, which permits unrestricted use, distribution, and reproduction in any medium, provided the original work is properly cited.

\begin{abstract}
Non-compliance is a common problem in chronic diseases including psychiatric disorders. Various attempts have been made for improving adherence in psychiatry; one of them being simplifying dosage regimen by designing modified dosage formulation. For example, depot formulation, oral disintegrating tablets or extended release formulations are some of the strategies for improving compliance in psychiatry patients. Modified dosage formulations also have some other advantages too. For example, oral disintegrating tablet of olanzapine cause lesser weight gain compared to standard olanzapine tablet while Zolpidem extended release tablet provides better sleep maintenance in middle of night. This article reviews some of the modified dosage formulations in psychiatric diseases with their advantages.
\end{abstract}

Keywords: Psychiatry, Dosage form, Compliance, Advantages

\section{INTRODUCTION}

Non-adherence to medicine is an issue in psychiatry. High rates of non-compliance have been reported in psychiatric disorders; $28 \%$ - 52\% for major depressive disorder, $20 \%-50 \%$ for bipolar disorder, and $20 \%-72 \%$ for schizophrenia. ${ }^{1}$ Non-compliance is one of the commonest causes of failure to achieve desired therapeutic response. It is not surprising to have more non-compliance in psychiatric diseases compared to other medical conditions. The issue of non-compliance in psychiatry diseases has been discussed since many decades. In one of the articles published more than 30 years before Seltzer A and Hoffman BF (1980) discussed compliance issues in psychiatric patients. In this article authors have discussed various reasons of noncompliance in psychiatry patients and also suggested some measures for improvement of compliance. Simplification of dosage regimen is one of the suggested measures in improving adherence in psychiatry patient.

Even today despite of having many drugs and different formulations, non-compliance in psychiatry patients still continues. A recently published study (2013) from Qatar, examined the prevalence and reasons for non-compliance in psychiatry out patients. Out of 564 patients, $41.8 \%$ were found to be non-compliant. Common diseases reporting non-compliance were schizophrenia (28.4\%), depression (14.4\%), and bipolar affective disorder $(12.7 \%)$. Poor family support, irregular attendance to clinic, ignorance about side effects of drugs, free medicines, and a lack of education about drug were commonly reported reasons for non-compliance in these patients. $^{3}$

Simple regimen and modified dosage forms help to improve patient compliance and adherence to treatment. They also help to improve patient outcome. In management of psychiatric diseases various modified release formulations are used; some of them have objective of improving patient compliance while others have different advantages. Modified drug delivery systems used in psychiatric disorders and their advantages are discussed below.

\section{MODIFIED DRUG FORMULATION IN BIPOLAR DISORDER}

\section{Divalproex sodium extended release tablets}

Divalproex is available in delayed release as well as extended release formulation. Delayed release and extended release formulations are not bioequivalent. For conversion from delayed release to extended release 
formulation, dose of extended release may need to be increased between $8 \%$ - $20 \%$ for achieving equivalent serum concentration. The extended-release tablet of divalproex sodium provides advantages of a once-daily administration. Other advantage of the extended release divalproex sodium is controlled and continued release of divalproex sodium from the tablet. Extended release formulation results in less fluctuation in the peak serum concentration compared to delayed release formulation. ${ }^{4}$

\section{MODIFIED DOSAGE FORMULATIONS SCHIZOPHRENIA}

IN

\section{Olanzapine mouth dissolving tablets}

Olanzapine is an effective and well-tolerated atypical antipsychotic used in the treatment of schizophrenia. Orally disintegrating olanzapine is available as an alternative to standard oral tablets of olanzapine. Orally disintegrating tablet rapidly disintegrates and dissolves in saliva. This formulation is convenient to patient and helps to enhance adherence to treatment. Patient preference and reduction in nursing burden are the other advantages with oral disintegrating tablets of olanzapine. ${ }^{5}$

Limitations for use of olanzapine include risk of the adverse events like weight gain, dyslipidemia and diabetes. Olanzapine oral disintegrating tablets have an advantage over standard tablet in this regard. Oral disintegrating tablets of olanzapine cause less weight gain. An open label prospective study in India determined the change in weight after switching from olanzapine standard tablet to olanzapine mouth dissolving tablet in 30 patients. These patients were switched to equivalent doses of oral disintegrating tablets from oral standard tablet of olanzapine. Evaluation of weight was done at baseline and at 4, 8, and 12 weeks. Following figure (Figure 1) shows $\%$ of patients with weight changes. ${ }^{6}$ The mean loss of weight reported was $1.9 \pm 0.3 \mathrm{~kg}$.

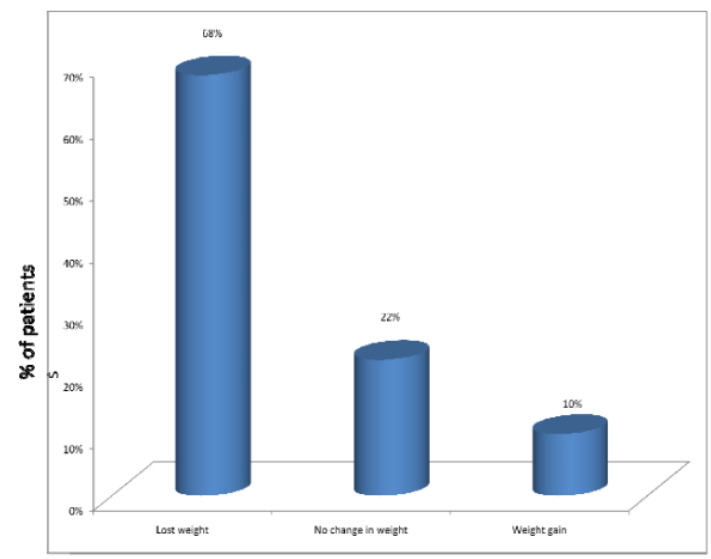

Figure 1: Percentages of patients with weight changes.

\section{Paliperidone extended release}

Schizophrenic patients lack insight. This is one of the challenges in the effective treatment and one of the causes for nonadherence in schizophrenia. Due to these reasons there is always an interest in developing drugs with once daily dosage or depot preparation with objective of improving adherence to treatment. Paliperidone is active metabolite of risperidone and available as paliperidone extended-release formulation. ${ }^{7}$

\section{Risperidone depot formulation}

Long-acting depot formulation of the second-generation antipsychotic drug risperidone is available for the treatment of schizophrenia. With this formulation, the treatment regimen for risperidone is simplified. Intramuscular depot injection of risperidone is administered every two weeks. ${ }^{8}$

\section{Asenapine sublingual tablet}

Asenapine is another antipsychotic used in the treatment of schizophrenia. It is not available as standard oral tablet. Asenapine is available as a sublingual fast dissolving tablet. The challenge in developing oral tablet of asenapine is it's high hepatic first pass metabolism 9 . Sublingual administration with asenapine is required because bioavailability is very less if it is ingested $(<2 \%)$. ${ }^{10}$

\section{MODIFIED DOSAGE FORMULATION FOR INSOMNIA MANAGEMENT}

\section{Zolpidem extended release formulation for treatment of Insomnia}

Patients suffering from Insomnia have difficulty in initiation or maintenance of sleep or wake up very early or may have combination of these symptoms. Zolpidem is $\mathrm{GABA}_{\mathrm{A}}$ receptor agonist which preferentially binds to alfa 1 subunit of receptor complex. It is used in short term management of Insomnia. Zolpidem extended release tablet is a dual-layer tablet. One layer releases zolpidem immediately while other layer releases it slowly. The objective of designing this formulation is treatment of both sleep onset and sleep maintenance insomnia. Zolpidem extended release $12.5 \mathrm{mg}$ tablet provides fast onset of action which helps for sleep induction but helps in better maintenance of sleep in middle of night. The profile of adverse events is similar to immediate release zolpidem tablet. ${ }^{11}$ Thus in zolpidem extended release formulation the objective of modified drug release is not improving compliance, but to provide better sleep maintenance in middle part of the night. A Multicenter, randomized, double-blind, placebo-controlled, study with zolpidem extended-release $12.5 \mathrm{mg}$ up to 6 months showed sustained and significant improvements in both sleep onset as well as sleep maintenance. ${ }^{12}$ 


\section{CONCLUSION}

Non-adherence to medicines is a one of the challenges in management of psychiatric disorders. Various strategies are used for increasing adherence to medical treatment; one of them being using simplified dosage regimen. Drug formulation modifications like extended release formulation, depot formulation, mouth dissolving formulation help to improve patient compliance. In addition to improving adherence, some formulations also have other advantages which help to improve outcome of treatment in psychiatric diseases.

\section{Funding: None}

Conflict of interest: None declared

Ethical approval: Not required

\section{REFERENCES}

1. Magura S, Rosenblum A, Fong C. Factors associated with medication adherence among psychiatric outpatients at substance abuse risk. The Open Addiction Journal 2011;4:58-64.

2. Seltzer A and Hoffman BF. Drug compliance of the psychiatric patient. Can Fam Physician 1980;26: 725-7.

3. Bener A, Dafeeah EF, Salem MO. A study of reasons of non-compliance of psychiatric treatment and patients' attitudes towards illness and treatment in Qatar. Issues in Mental Health Nursing. April 2013;34:273-80.

4. Stoner SC, Dahmen MM. Extended-release divalproex in bipolar and other psychiatric disorders: Acomprehensive review. Neuropsychiatric Disease and Treatment 2007;3(6):839-46.
5. Montgomery W, Treuer T, Karagianis J, AscherSvanum H, Harrison G. Orally disintegrating olanzapine review: effectiveness, patient preference, adherence, and other properties. Patient Preference and Adherence 2012;6:109-25.

6. Shukla RK, Sarkar P, Mishra A. Weight loss after switching to olanzapine mouth dissolving tablets-An open label prospective study. Orissa Journal Psychiatry 2009;27-30.

7. Gahr M, Kölle MA, Schönfeldt-Lecuona C, Lepping P, Freudenmann RW. Paliperidone extendedrelease: does it have a place in antipsychotic therapy? Drug Design, Development and Therapy 2011;5:125-46.

8. Rainer MK. Risperidone long-acting injection: a review of its long term safety and efficacy. Neuropsychiatric Disease and Treatment 2008;4(5):919-27.

9. Balaraman R, Gandhi H. Asenapine, a new sublingual atypical antipsychotic. J Pharmacol Pharmacother 2010;1(1):60-1.

10. Citrome L. Role of sublingual asenapine in treatment of schizophrenia. Neuropsychiatr Dis Treat 2011;7:325-39.

11. Moen MD, Plosker GL. Zolpidem extended release. CNS Drugs 2006;20(5):419-26.

12. Krystal AD, Erman M, Zammit GK, Soubrane C, Roth T. Long-Term Efficacy and Safety of Zolpidem Extended-Release $12.5 \mathrm{mg}$, Administered 3 to 7 Nights Per Week for 24 Weeks, in Patients With Chronic Primary Insomnia: A 6-Month, Randomized, Double- Blind, Placebo-Controlled, Parallel-Group, Multicenter Study. Sleep 2008;31:79-90.

doi:10.5455/2319-2003.ijbcp20130804

Cite this article as: Patil AD. Advantages of modified drug delivery system in psychiatry. Int $\mathbf{J}$ Basic Clin Pharmacol 2013;2:368-70. 